\title{
Increased expression of endothelial antigen PAL-E in human diabetic retinopathy correlates with microvascular leakage
}

\author{
R.O. Schlingemann ${ }^{1}$, P. Hofman ${ }^{1,3}$, G. F. J.M. Vrensen ${ }^{2,3}$, H. G. T. Blaauwgeers ${ }^{1,3}$ \\ ${ }^{1}$ Department of Ophthalmology, Academic Medical Centre, University of Amsterdam, Amsterdam, The Netherlands \\ ${ }^{2}$ Department of Ophthalmology, Leiden University Medical Centre, Leiden, The Netherlands \\ ${ }^{3}$ Department of Morphology, Netherlands Ophthalmic Research Institute, Amsterdam, The Netherlands
}

\begin{abstract}
eyes of diabetic patients and in 2 of the 30 normal Aims/hypothesis. The Pathologische Anatomie Leiden-Endothelium (PAL-E) antigen is a marker for loss of the blood-brain barrier function in brain tumours. It is endothelium specific and is associated with the endothelial plasmalemmal vesicles (caveolae) involved in transcellular transport. To test whether blood-retinal barrier loss in diabetic retinopathy is associated with cellular changes in the endothelium, the expression of antigen PAL-E in relation to microvascular leakage in human diabetic retinopathy was investigated.

Methods. Immunohistochemical staining of frozen tissue sections of postmortem eyes obtained from 30 persons without and 41 persons with diabetes mellitus was carried out with monoclonal antibodies against PAL-E and CD31 and with antibodies against endogenous fibrinogen, albumin and $\mathrm{IgG}$ as indicators of vascular leakage.

Results. Patchy or uniform microvascular PAL-E staining was observed in the retina of 17 of the 41

control eyes. In the diabetic eyes, PAL-E staining co-localized with microvascular staining for endogenous fibrinogen, albumin and IgG. Strong staining for PAL-E was observed in sites without blood-tissue barriers, like the choroid.

Conclusions/interpretation. In microvessels with an intact blood-retina barrier the endothelial antigen PAL-E is absent. Its expression is increased in retinal vessels of patients with diabetic retinopathy and correlates with microvascular leakage of plasma proteins. This phenotypic shift involving an antigen associated with caveolae suggests that dysfunction of the endothelium forms the cellular basis for microvascular leakage in diabetic retinopathy, rather than passive endothelial damage. [Diabetologia (1999) 42: 596-602]

Keywords Diabetic retinopathy, diabetes mellitus, blood-retinal barrier, capillary permeability, vascular endothelium, caveolae, immunohistochemistry, fibrinogen.
\end{abstract}

Changes in the vascular blood-retina barrier are important in the course of diabetic retinopathy, as they give rise to leakage and ultimately lead to visual loss

Received: 10 September 1998 and in final revised form: 26 November 1998

Corresponding author: R. O. Schlingemann, M.D., PhD., Department of Ophthalmology, Academic Medical Centre, University of Amsterdam, Meibergdreef 9, 1105 AZ Amsterdam, The Netherlands

Abbreviations: PAL-E, Pathologische Anatomie Leiden-Endothelium; VEGF, vascular endothelial growth factor; $\mathrm{mAb}$, monoclonal antibody; FA, fluorescein angiography. in many patients [1-4]. The mechanisms by which these changes occur, and whether mediators such as growth factors are involved, are largely not known [2-8]. The role of endothelial cells is also unclear. The leakage may be a passive process due to metabolic compromise and decompensation of the endothelium or could be due to a dysfunction of the endothelium, possibly caused by growth factors [1-8]. Recent reports have shown expression of vascular endothelial growth factor (VEGF) near leaky vessels in human and experimental diabetic retinopathy [9-12], suggesting a role of VEGF as a permeability factor in this disease. 
Table 1. Clinical data and degree of retinal vascular PAL-E staining in eyes from diabetic patients obtained at autopsy

\begin{tabular}{|c|c|c|c|c|c|c|c|c|}
\hline $\begin{array}{l}\text { Autopsy } \\
\text { number }\end{array}$ & $\begin{array}{l}\text { Ophthalmological } \\
\text { history }\end{array}$ & Age & FA & $\begin{array}{l}\text { Duration of } \\
\text { Type II diabetes } \\
\text { mellitus }\end{array}$ & Cause of death & Findings at autopsy & Retinopathy & $\begin{array}{l}\text { PAL-E } \\
\text { staining }\end{array}$ \\
\hline $1-1993$ & pseudophakia OD & 83 & - & 13 years & pneumonia & no nephropathy & $\begin{array}{l}\text { few MA*, } \\
\text { no leakage } \\
(4-1993)\end{array}$ & none \\
\hline $2-1993$ & - & 90 & - & $?$ & $\begin{array}{l}\text { myocardial } \\
\text { infarction }\end{array}$ & no nephropathy & $?$ & sporadic \\
\hline $4-1993$ & - & 71 & - & $?$ & pneumonia & early nephropathy & $?$ & sporadic \\
\hline $5-1993$ & pseudophakia OS & 75 & - & 9 years & $\begin{array}{l}\text { myocardial } \\
\text { infarction }\end{array}$ & $\begin{array}{l}\text { diabetic fibrosis of } \\
\text { pancreas and athe- } \\
\text { rosclerosis }\end{array}$ & $\begin{array}{l}\text { none } \\
(3-1987)\end{array}$ & sporadic \\
\hline 6-1993 & - & 73 & - & $?$ & unknown & no nephropathy & $?$ & none \\
\hline 9-1994 & - & 57 & - & $?$ & ALS* & no nephropathy & $?$ & none \\
\hline 10-1994 & $\begin{array}{l}\text { cataract, AMD* } \\
\text { and glaucoma }\end{array}$ & 73 & +1993 & 31 years & $\begin{array}{l}\text { myocardial } \\
\text { infarction }\end{array}$ & early nephropathy & $\begin{array}{l}\text { background DRP } \\
\text { focal leakage }\end{array}$ & uniform \\
\hline
\end{tabular}

FA = fluorescein angiography; DRP = diabetic retinopathy; ?= unknown; MA = microaneurysms; ALS = amyotrophic lateral sclerosis; $\mathrm{AMD}=$ age-related macular degeneration; $\mathrm{OD}=$ right eye; $\mathrm{OS}=$ left eye

It was shown previously that the distribution of the Pathologische Anatomie Leiden-Endothelium (PALE) antigen [13] in brain and eye is restricted to sites where no barrier function is present, e.g. dura, choroid plexus, area postrema and choroid, and to pathological conditions characterized by absence of a barrier as in primary and metastatic brain tumours [14-17]. In other parts of the brain and eye where the blood-tissue barriers are present, this endothelium-specific antigen is absent [14-17]. At the electron microscopic level the antigen is associated with the plasmalemmal vesicles (caveolae) of endothelial cells $[13,18]$. These caveolae have a function in transcellular transport and are scare in the endothelium in areas with a blood-tissue barrier [19-22]. The PAL-E antigen therefore may have a function in non-specific trancellular transport [13-17]. We did an immunohistochemical study in eye tissues from diabetic patients and normal control subjects to test the hypotheses that 1) the PAL-E antigen is involved in vascular permeability, 2) that endothelial PAL-E staining may indicate barrier loss in pre-existent vessels in the retina and 3) that vascular leakage in diabetic retinopathy involves endothelial cellular changes.

\section{Materials and methods}

Tissue samples. The investigations were done in accordance with the principles of the Declaration of Helsinki. Human eyes from 30 donors (age range 28 to 88 years, mean 70 years) with no known history of diabetes mellitus were kindly provid- ed by the Corneabank Amsterdam, In addition, eyes of 41 people with known diabetes mellitus (age range 48 to 90 years, mean 75 years) were obtained from the Corneabank Amsterdam $(n=31)$ or enucleated at autopsy at the Department of Pathology, Academic Medical Centre, Amsterdam $(n=10)$. The cause of death of the cornea donors was known, but for reasons of privacy information on the type of diabetes and the history of eye disease, in particular of the posterior segment, was unavailable. Relevant clinical data of the autopsy patients are listed in Table 1. The ophthalmological history was available for five of these patients and for two a fluorescein angiogram (FA) was available. Whole eyes were snap-frozen in isopenthane within 3 to $28 \mathrm{~h}$ (mean $16 \mathrm{~h}$; diabetic patients) and 5 to $36 \mathrm{~h}$ (mean $19 \mathrm{~h}$; control subjects) after death of the donor. In all cases identical samples of the posterior segment were dissected and stored at $-70^{\circ} \mathrm{C}$.

Immunoperoxidase staining. Cryo-sections of samples were air-dried, fixed with acetone for $10 \mathrm{~min}$ and postfixed with Zamboni fixative ( $2 \%$ paraformaldehyde in a saturated picric acid solution). Serial sections were stained by an indirect immunoperoxidase procedure with the following antibodies: monoclonal antibodies (mAbs) PAL-E (Pathologische Anatomie Leiden-Endothelium) [13] and the general endothelial marker mAb EN-4 (anti-CD 31) (Mon 6002, Sanbio, Uden, The Netherlands) [18]. To analyse vascular leakage, sections were stained with a MoAb reactive with human fibrinogen (kindly provided by Dr. J.J. Emeis from the Gaubius Laboratoriurn TNO/PG, Leiden, The Netherlands) [23], an antibody reactive with human $\mathrm{IgG}$ (polyclonal goat anti-human $\mathrm{IgG}$ conjugated to horseradish peroxidase) and a rabbit polyclonal antibody reactive with human albumin (DAKO, Copenhagen, Denmark) [5-9, 24]. All antibodies were used at appropriate dilutions and goat anti-mouse $\mathrm{IgG}$ and swine anti-rabbit $\mathrm{IgG}$ conjugated to horseradish peroxidase (DAKO, Copenhagen, Denmark) were used as secondary antibodies. For staining, incubation for $10 \mathrm{~min}$ in 3-amino-9-ethylcarbazole containing 

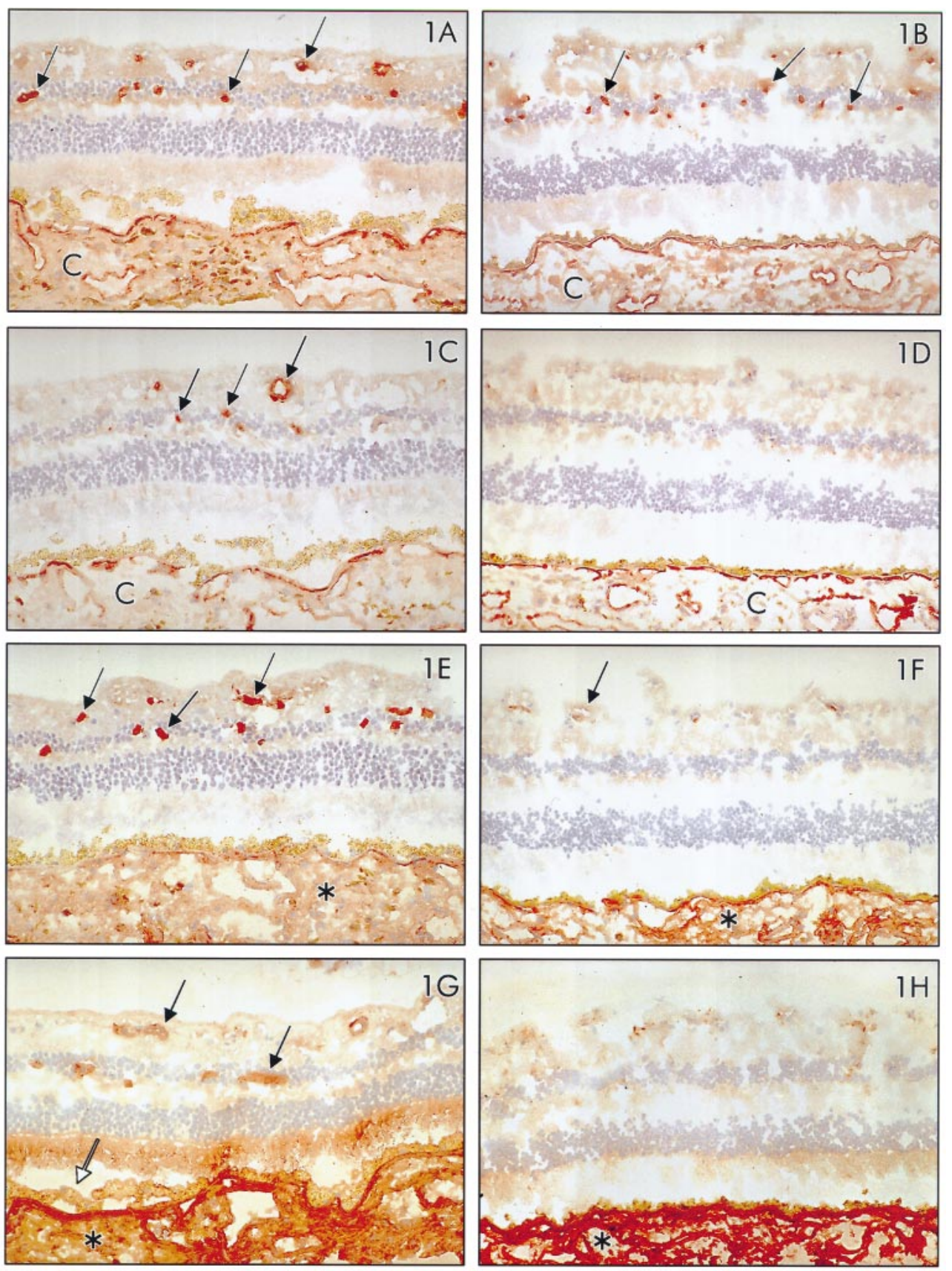

Fig. 1 A-H Immunoperoxidase staining of frozen tissue sections of an area of retina of a diabetic patient (left panels) and a non-diabetic control donor (right panels) with anti-CD31 $(\mathbf{A}, \mathbf{B})$, anti-PAL-E $(\mathbf{C}, \mathbf{D})$, anti-fibrinogen $(\mathbf{E}, \mathbf{F})$, and anti$\operatorname{IgG}(\mathbf{G}, \mathbf{H})$. (Note pronounced staining of all microvessels for CD31 (A, B), but absent staining of the capillaries in the normal retina for PAL-E $(\mathbf{D})$, anti-fibrinogen $(\mathbf{F})$, and anti-IgG (H). In the retina of the diabetic patient, staining with anti-fibrinogen $(\mathbf{E})$, and anti-IgG $(\mathbf{G})$ indicates leaking capillaries, which are also stained for PAL-E (C) (capillaries indicated by arrows; $\mathrm{C}=$ choroid). Note the diffuse stromal staining in the choroid for fibrinogen and $\mathrm{IgG}$ and staining of the choroidal vasculature by anti-PAL-E in both sections $(\mathbf{C}-\mathbf{D})$, (staining indicated by asterisks $)(\times 120)$

$0.01 \% \mathrm{H}_{2} \mathrm{O}_{2}$ was used [25]. In negative control sections the first antibodies were omitted. Counterstaining was done with haematoxylin. All sections were examined by two independent observers and scored blind. Distribution of the staining product and intensity of staining were assessed for all antibodies. Distribution of retinal vascular staining for PAL-E and fibrinogen was graded semi-quantitatively as follows: 0 , no staining; 1 , sporadic staining (less than three positive capillaries per $5 \mathrm{~mm}$ section); 2, patchy staining (localized areas with staining capillaries, but less than $50 \%$ of vessels positive); or 3 , uniform staining (more than $50 \%$ of vessels positive). For the correlation between the two ordinal variables PAL-E staining and fibrinogen staining, we used the conventional non-parametric rank-order gamma-test, which can be interpreted as a correlation coefficient (SPSS version 8.0, section Crosstabs, SPSS Inc., USA) [26]. 


\section{Results}

Of the retinas of donors with diabetes mellitus, 17 of 41 showed pronounced microvascular staining with PAL-E in a patchy or uniform distribution whereas in 8 a weak or pronounced sporadic staining of retinal capillaries was observed (Fig. 1C and 2C). The PAL$\mathrm{E}$ positive vessels, localized in both the superficial and deep inner retina, were often larger than vessels in normal retinas. In some instances, the endothelium of a large retinal vessel was stained at the side facing the neuroretina (results not shown). Four of the PAL-E positive eyes showed microaneurysms or hard exudates or both (Fig. 2D), exclusively observed in the PAL-E positive areas.

In serial sections, staining for PAL-E was compared with sections stained with anti-fibrinogen (all eyes), anti-IgG (in ten diabetic and ten control eyes), anti-albumin (in ten diabetic and ten control eyes) and the pan-endothelial marker EN-4 (CD 31) (all eyes). Staining for CD31 was observed in all retinal vessels in both control and diabetic eyes, and this enabled grading of the extent of vascular staining of PAL-E and anti-fibrinogen. Staining for endogenous fibrinogen was patchy or uniform in the retina of 14 out of 41 diabetic eyes and sporadic in 9. Reaction product was confined to the microvascular walls and did usually not extend to the perivascular area (Figs. $1 \mathrm{~B}$ and 2D). In almost all eyes, serial sections indicated that positive staining for fibrinogen and PAL-E colocalized in the same microvessels in corresponding areas (Figs. 1 and 2) (Table 2). This correlation was high for the two groups combined (gamma $=0.753$, $p<0.001$ ). In the ten diabetic patients studied, staining for IgG and albumin was also observed in the areas with PAL-E positive vessels, both in the vascular walls and in a more diffuse pattern (Fig. $1 \mathrm{G}$ ).

In a small number of the donors and autopsy patients, clinical information was available about the presence and degree of diabetic retinopathy or other diabetic complications (summarized for the autopsy patients in Table 1). One donor with uniform pronounced PAL-E staining had been treated with laser for proliferative diabetic retinopathy. Another patient with uniform marked PAL-E staining (autopsy patient 10 , Table 1 ) suffered clinically from diabetic retinopathy with a fundus picture of scattered dot haemorrhages, a few hard exudates and microaneurysms. Focal leakage in the posterior pole of this eye was shown by fluorescein angiography (FA) carried out 6 months before death. In addition to the pronounced PAL-E staining within the retina, this eye histologically showed proliferative retinopathy with preretinal neovascularization consisting of PAL-E positive capillaries (results not shown). Another diabetic patient with a patchy distribution of PAL-E staining (autopsy patient 7) had early background diabetic retinopathy 4 years before death, with a few
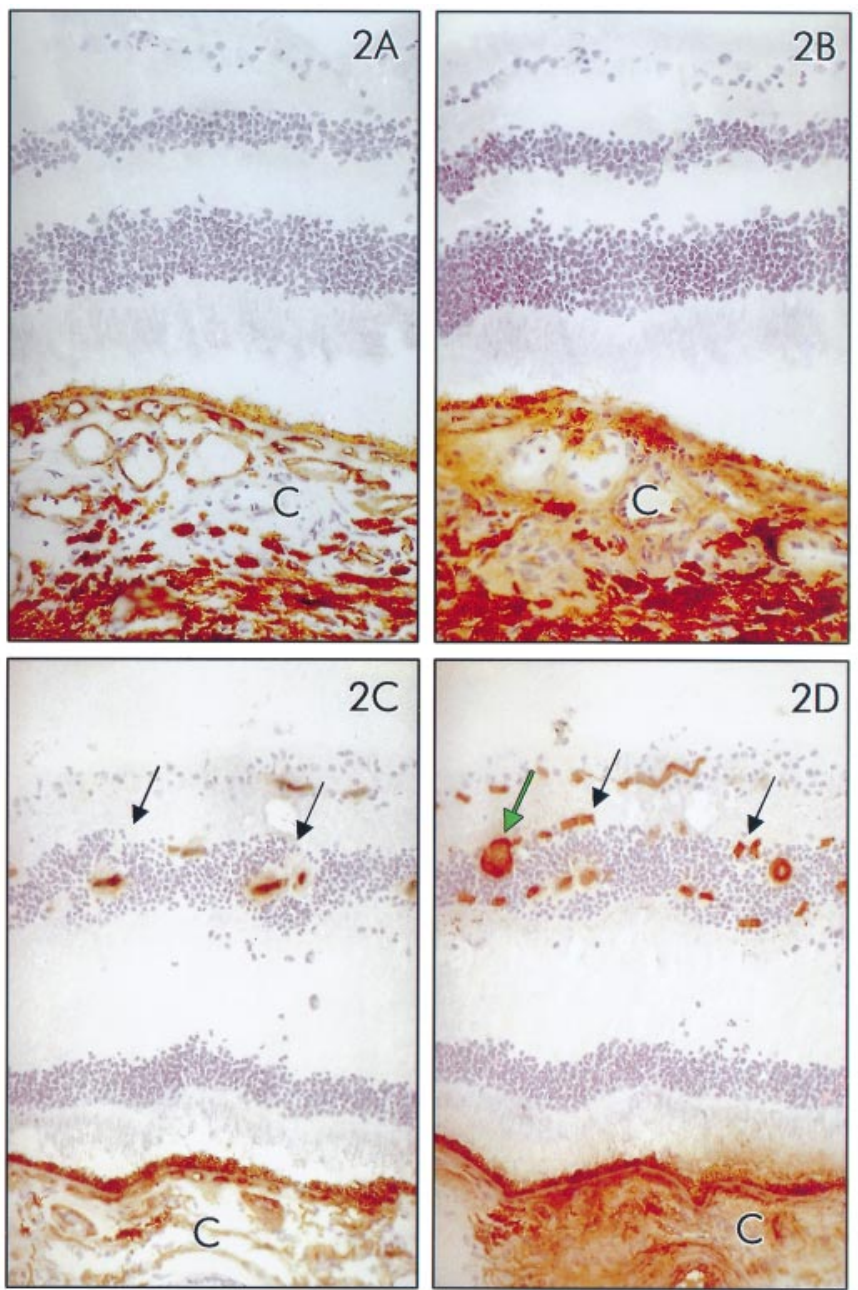

Fig. 2 A-D Immunoperoxidase staining of serial frozen tissue sections of retina of a diabetic patient (lower panels) and a non-diabetic control donor (upper panels) with anti-PAL-E $(\mathbf{A}, \mathbf{C})$ and anti-fibrinogen $(\mathbf{B}, \mathbf{D})$. No staining for PAL-E or fibrinogen is observed in the retina of the non-diabetic person $(\mathbf{A}, \mathbf{B})$. Note pronounced staining of retinal microvessels with both anti-fibrinogen $(\mathbf{D})$ and anti-PAL-E $(\mathbf{C})$ in the diabetic patient (capillaries indicated by arrows), and the extensive staining of the capillary walls by anti-fibrinogen (D). A microaneurysm stained with anti-fibrinogen is seen in the inner nuclear layer $(\mathbf{D}$, green arrow) $(\mathrm{C}=$ choroid $)(\times 180)$

scattered microaneurysms and minimal leakage shown by FA (Table 1) (results not shown).

In 21 out of 30 control eyes, retinal capillaries were completely negative for PAL-E (Figs. 1D and 2A), but in 7 eyes sporadic, and in 2 eyes a patchy staining was observed (Table 2). In all of these eyes staining intensity was weak. Sporadic staining for fibrinogen was observed in eight normal eyes and in two eyes a patchy distribution was seen. In these ten eyes fibrinogen staining intensity was weak and mainly intravascular (Fig. 1F) (results summarized in Table 2). Retinal staining for $\mathrm{IgG}$ and albumin was absent in the control subjects, except for weak staining for $\mathrm{IgG}$ in the photoreceptor layer in most patients (Fig. $1 \mathrm{H})$. 
Table 2. Summary of immunohistochemical staining results and correlation of the distribution of mAb PAL-E and anti-fibrinogen staining in retina of eyes of diabetic patients and control subjects

\begin{tabular}{|c|c|c|c|c|c|c|c|}
\hline \multirow[t]{2}{*}{ Group } & & \multirow[t]{2}{*}{ Staining type } & \multicolumn{4}{|c|}{ Anti-fibrinogen staining } & \multirow[t]{2}{*}{ Total } \\
\hline & & & Absent & Sporadic & Patchy & Uniform & \\
\hline \multirow{4}{*}{$\begin{array}{l}\text { Diabetic } \\
\text { patients }\end{array}$} & PAL-E & Absent & 13 & 3 & & & 16 \\
\hline & & Patchy & 1 & 3 & 7 & & 11 \\
\hline & & Uniform & & & & 6 & 6 \\
\hline & & Total & 18 & 9 & 8 & 6 & 41 \\
\hline \multirow{3}{*}{$\begin{array}{l}\text { Control } \\
\text { subjects }\end{array}$} & & Patchy & 2 & & & & 2 \\
\hline & & Uniform & & & & & \\
\hline & & Total & 20 & 8 & 2 & & 30 \\
\hline
\end{tabular}

(correlation coefficient for the two groups combined, gamma $=0.753, p<0.001$ )

\section{Discussion}

In this study we show that microvascular leakage in the retina of patients with diabetes mellitus, identified by immunohistochemical staining for endogenous fibrinogen, $\operatorname{IgG}$ or albumin, is associated with increased expression of the endothelial antigen PAL-E in the retinal microvasculature.

Because of the absence of case histories of most of the donors, our immunohistochemical findings could not be correlated to the clinical degree of diabetic retinopathy or type of diabetes. Where histological evidence of diabetic retinopathy in the studied tissue sections or clinical information on the presence of diabetic retinopathy or other diabetic complications were available, however, these data supported a correlation between the degree of diabetic retinopathy and the immunohistochemical evidence of PAL-E and fibrinogen-positive retinal vessels. In fact, pronounced uniform staining of intraretinal vessels with PAL-E was found in the two patients with known proliferative diabetic retinopathy. The function and molecular characteristics of the PAL-E antigen are not known [13-16]. Still, its tissue distribution and ultrastructural localization are of considerable interest [13-17]. In the brain, eye and testis, the endothelium of vessels constituting a blood-tissue barrier lacks the antigen, whereas permeable capillaries and veins in other organs and in the so-called "special sites" in the brain, like dura, area postrema and choroid plexus, have pronounced expression [13-17]. In both cultured endothelial cells and in vivo the antigen is specifically localized in pinocytotic plasmalemmal vesicles or caveolae $[13,18]$. These organelles play a major part in trans-endothelial transport and are scarce in blood-tissue barrier endothelia [19-22]. In brain tumours, where considerable barrier loss is seen, the endothelial cells have increased numbers of pinocytotic vesicles and substantially increased expression of PAL-E $[14,15]$, supporting the association of the
PAL-E antigen with trans-endothelial transport via pinocytotic vesicles. The results of this study show a direct correlation between the presence of increased PAL-E expression by barrier endothelial cells and pathological vascular leakage of plasma proteins, lending further support for a role of this antigen in non-specific transendothelial transport. This study also indicates that the PAL-E antigen is useful as a marker for leaky capillaries in eye disease [24].

In the diabetic eyes studied here, retinal vascular PAL-E expression correlated with staining for endogenous fibrinogen $[8,23]$ and, in a smaller series of eyes, with vascular and perivascular staining for albumin and IgG. Perivascular staining for fibrinogen in the diabetic rat and human retina has been used previously as an indicator of increased permeability $[8$, 24]. Surprisingly, we found little or no perivascular staining for fibrinogen in the eyes of diabetic patients, but pronounced staining of the entire capillary wall in contrast with the weak intravascular staining observed in the eyes of control subjects. This pattern could represent structural changes in the microvascular wall and basement membrane in the diabetic eye $[2,3]$. Our results indicate that immunohistochemical staining for PAL-E and fibrinogen provides a new and useful means to identify areas affected by diabetic retinopathy in histological sections of human retina [24].

These findings may have implications for the understanding of the mechanism by which blood-retinal barrier loss occurs in diabetic retinopathy. In view of its specific localization in pinocytotic vesicles, the increased expression in the diabetic retina of the endothelial antigen PAL-E suggests an active role of endothelial cells in the pathogenesis of diabetic retinopathy. This supports the notion that pathological blood-retinal barrier loss in diabetic retinopathy is caused by altered endothelial function. In the normal retina, the blood-retinal barrier is formed by endothelial cells with properties similar to brain endotheli- 
um $[27,28]$. The cells have tight junctions, few pinocytotic vesicles and express a number of specific molecules on their surface that are associated with transport of glucose and other nutrients [20, 21, 24, $25,28]$. In many diabetic patients, a diffuse increased permeability of the blood-ocular barriers can be determined by vitreous fluorophotometry, before the development of clinical diabetic retinopathy or visible retinal abnormalities on fluorescein angiography (FA) [1]. In clinical diabetic retinopathy, retina oedema and exudates are observed with characteristic focal or diffuse retinal extravasation of fluorescein on FA [1]. It is not known whether different mechanisms underlie these two types of increased permeability of the blood-ocular barriers in diabetes mellitus. The "leakage" of retinal vessels may involve opening of the endothelial tight junctions or increased pinocytotic transport [1, 4-6]. As we observed widespread vascular staining for the PAL-E antigen in the retina of $41 \%$ of our diabetic patients, particularly in those with known diabetic retinopathy, this phenotypic shift could be associated primarily with the clinically meaningful second type of "leakage," a situation that could well be accompanied by an endothelial dysfunction more severe than in the early preclinical stages.

Our findings are particularly interesting in view of the role of vascular permeability factor/vascular endothelial growth factor (VEGF) in the pathogenesis of diabetic retinopathy $[9-12,29]$, as this factor has been shown to enhance vascular permeability in in vivo models [12] and induce increased numbers of endothelial vesicles in vitro [30] and in vivo [31]. VEGF appears to be an important angiogenesis factor in proliferative diabetic retinopathy and neovascularization of the iris [12]. A number of reports suggested that VEGF is also involved in retinal vascular leakage in background diabetic retinopathy in rats and humans [9-11]. A recent paper questioned these results, however, indicating that VEGF protein and its messenger RNA are also present [29] in the normal retina. The relation between PAL-E expression and VEGF will be addressed in further studies.

Acknowledgements. This study was supported by the Diabetes Fonds Nederland (Grant 95.103), the Haagsch Oogheelkundig Fonds, the Edward and Marianne Blaauwfonds and the Landelijke Stichting voor Blinden en Slechtzienden. The authors wish to thank the Department of Pathology, Academic Medical Centre, Amsterdam, The Netherlands (Head Prof. Dr. J.J. Weening), the BIS Foundation (Leiden, The Netherlands) and the Corneabank (Amsterdam, The Netherlands, Head Dr. E. Pels) for their assistance in obtaining the tissues, Dr. J.J Emeis from the Gaubius Laboratorium TNO/PG, Leiden, The Netherlands, for providing the antibody against fibrinogen, Dr. G.Bonsel for the statistical analysis, and N. Bakker, M. Danzman, and T. Put for preparing the microphotographs.

\section{References}

1. Cunha-Vaz JG (1983) Studies on the pathophysiology of diabetic retinopathy. The blood-retinal barrier in diabetes. Diabetes 32 [Suppl]:20-27

2. Ruggiero D, Lecomte M, Michoud E, Lagarde M, Wiernsperger N (1997) Involvement of cell-cell interactions in the pathogenesis of diabetic retinopathy. Diabetes Metab 23: $30-42$

3. Porta M (1996) Endothelium: the main actor in the remodelling of the retinal microvasculature in diabetes. Diabetologia 39: 739-744

4. Cunha-Vaz JG (1980) Blood-retinal barriers in health and disease. Trans Ophthalmol Soc UK 100: 337-340

5. Vinores SA, McGehee R, Lee A, Gadegbeku C, Campochiaro PA (1990) Ultrastructural localization of blood-retinal barrier breakdown in diabetic and galactosemic rats. $\mathbf{J}$ Histochem Cytochem 38: 1341-1352

6. Vinores SA, Van Niel E, Swerdloff JL, Campochiaro PA (1993) Electron microscopic immunocytochemical demonstration of blood-retinal barrier breakdown in human diabetics and its association with aldose reductase in retinal vascular endothelium and retinal pigment epithelium. Histochem J 25: 648-663

7. Murata T, Ishibashi T, Inomata H (1993) Immunohistochemical detection of blood-retinal barrier breakdown in streptozotocin-diabetic rats. Graefes Arch Clin Exp Ophthalmol 231: 175-177

8. Murata T, Ishibashi T, Inomata H (1992) Immunohistochemical detection of extravasated fibrinogen (fibrin) in human diabetic retina. Graefes Arch Clin Exp Ophthalmol 230: 428-431

9. Murata T, Nakagawa K, Khalil A, Ishibashi T, Inomata H, Sueishi K (1996) The relation between expression of vascular endothelial growth factor and breakdown of the bloodretinal barrier in diabetic rat retinas. Lab Invest 74: 819-825

10. Lutty GA, McLeod DS et al. (1996) Localization of vascular endothelial growth factor in human retina and choroid. Arch Ophthalmol 114: 971-977

11. Amin RH, Frank RN et al. (1997) Vascular endotheliall growth factor is present in glial cells of the retina and optic nerve of human subjects with nonproliferative diabetic retinopathy. Invest Ophthalmol Vis Sci 38: 36-47

12. Schlingemann RO, van Hinsbergh VW (1997) Role of vascular permeability factor/vascular endothelial growth factor in eye disease. Perspective. Br J Ophthalmol 81: $501-512$

13. Schlingemann RO, Dingjan GM, Blok J, Emeis JJ, Warnaar SO, Ruiter DJ (1985) Monoclonal antibody PAL-E specific for endothelium. Lab Invest 52: 71-76

14. Schlingemann RO, Bots GTAM, VanDuinen SG, Ruiter DJ (1988) Differential expression of endothelium-specific antigen PAL-E in vasculature of brain tumors and preexistent brain capillaries. In: Strand F (ed) Fourth colloquium in biological sciences: Blood-brain transfer. Ann NY Acad Sci 529: 111-114

15. Leenstra S, Troost D, Das PK, Claessen N, Becker AE, Bosch DA (1993) Endothelial cell marker PAL-E reactivity in brain tumor, developing brain, and brain disease. Cancer 72: 3061-3067

16. Schlingemann RO, Hofman P, Andersson L, Troost D, Van der Gaag R(1997) Vascular expression of endothelial antigen PAL-E indicates absence of blood-ocular barriers in the normal eye. Ophthalmic Res 29: 130-138

17. Schlingemann RO, Hofman P, Klooster J, Blaauwgeers HGT, Van der Gaag R, Vrensen GFJM (1998) Ciliary mus- 
cle capillaries have blood-tissue barrier characteristics. Exp Eye Res 66: 747-754

18. Schlingemann RO, Rietveld FJR, DeWaal RMW, Ruiter DJ (1991) Differential expression of markers for endothelial cells, pericytes and basal lamina in the microvasculature of tumors and granulation tissue. Am J Pathol 138: 1335-1347

19. Wagner RC, Chen SC (1991) Transcapillary transport of solute by the endothelial vesicular system: evidence from thin serial sections analysis. Microvasc Res 42: 139-150

20. Schnitzert JE, Oh P, Pinney E, Allard J (1994) Filipin-sensitive caveolae-mediated transport in endothelium: reduced transcytosis, scavenger endocytosis, and capillary permeability of select macromolecules. J Cell Biol 127: 1217-1232

21. Bradbury MWB (1985) The blood-brain barrier: transport across the cerebral endothelium. Circ Res 57: 213-222

22. Rapaport SI (1976) Sites and function of the blood-brain barrier. In: Rapaport SI (ed) Blood brain barrier in physiology and medicine. Raven press, New York, pp 43-86

23. Koppert PW, Huijsmans CMG, Nieuwenhuizen W (1985) A monoclonal antibody, specific for human fibrinogen, fibrinopeptide A-containing fragments and not reacting with free fibrinopeptide A. Blood 66: 503-507
24. Vinores SA (1995) Assessment of blood-retinal barrier integrity. Histol Histopathol 10: 141-154

25. Graham RC, Lundholm U, Karnovsky MJ (1965) Cytochemical demonstration of peroxidase activity with 3-amino-9-ethylcarbazole. J Histochem Cytochem 13: 150-152

26. Siegel S, Castellan NJ (1988) Nonparametric statistics. 2nd edn. McGraw-Hill, New York, pp 291-298

27. Cunha-Vaz J (1979) The blood-ocular barriers. Surv Ophthalmol 23: 279-296

28. Raviola G (1977) The structural basis of the blood-ocular barriers. Exp Eye Res 25: 27-64

29. Gerhardinger C, Brown LF, Roy S, Mizutani M, Zucker CL, Lorenzi M (1998) Expression of vascular endothelial growth factor in the human retina and in non-proliferative diabetic retinopathy. Am J Pathol 152: 1453-1462

30. Esser S, Wolburg K, Wolburg H et al. (1998) Vascular endothelial growth factor induces endothelial fenestrations in vitro. J Cell Biol 140: 947-959

31. Feng D, Nagy JA, Hipp J, Dvorak BF, Dvorak AM (1996) Vesiculo-vacuolar organelles and the regulation of venule permeability to macromolecules by vascular permeability factor, histamine, and serotonin. J Exp Med 183: 1981-1986 